MADPH-95-888

astro-ph/9512080

University of Wisconsin - Madison

December 1995

\title{
Ultra-Transparent Antarctic Ice as a Supernova Detector
}

\author{
F. Halzen ${ }^{1}$, J.E. Jacobsen ${ }^{1}$ and E. Zas ${ }^{2}$ \\ ${ }^{1}$ Department of Physics, University of Wisconsin, Madison, WI 53706, USA \\ ${ }^{2}$ Dpto. de Partículas, Universidad de Santiago, E-15706 Santiago, Spain
}

\begin{abstract}
We have simulated the response of a high energy neutrino telescope in deep Antarctic ice to the stream of low energy neutrinos produced by a supernova. The passage of a large flux of $\mathrm{MeV}$-energy neutrinos during a period of seconds will be detected as an excess of single counting rates in all individual optical modules. We update here a previous estimate of the performance of such an instrument taking into account the recent discovery of absorption lengths of several hundred meters for near-UV photons in natural deep ice. The existing AMANDA detector can, even by the most conservative estimates, act as a galactic supernova watch.
\end{abstract}




\section{INTRODUCTION}

Although aspects of the observations of SN1987A [1.2] left some lingering doubts about supernova models [0, 1 , they provided, in general, remarkable confirmation of established ideas about supernova mechanisms [5]. At collapse, the core of the progenitor star is expected to release energy in a prompt $\nu_{e}$ burst lasting a few milliseconds. Most of the energy is, however, liberated after deleptonization in a burst lasting about ten seconds. Roughly equal energies are carried by each neutrino species with a thermal spectrum of temperature $2-4 \mathrm{MeV}$. The time scale corresponds to the thermalization of the "neutrinosphere" and its diffusion within the dense core [6].

Since the $\bar{\nu}_{e}$ cross-section [7] on protons in the detector is significantly larger than the interaction cross sections for the other neutrino flavors, $\bar{\nu}_{e}$ events dominate the signal by a large factor after detection efficiency is taken into account. In this reaction, free protons absorb the antineutrino to produce a neutron and a positron which is approximately isotropically emitted with an energy close to that of the initial neutrino. A thermal spectrum of temperature $4 \mathrm{MeV}$, when folded with an inverse beta decay cross section which increases with the square of the neutrino energy, yields an observed positron energy distribution which peaks in the vicinity of $20 \mathrm{MeV}$.

High energy neutrino telescopes under construction deploy Optical Modules (OM's) in a clear medium which acts as the radiator of Čerenkov light from muon tracks or electromagnetic showers produced in neutrino interactions. They are primarily designed to exploit the long range of high energy muons and have typical nominal threshold energies in the GeVrange, ostensibly too high to observe supernova-neutrinos. However, the interactions of $\bar{\nu}_{e}$ with protons produces copious numbers of positrons with tens of MeVs of energy. These will yield signals in all OMs during the (typically 10 second) duration of the burst. Such a signal, even if statistically weak in a single OM, will become significant for a sufficient number of OMs. The number of OMs required for monitoring galactic supernovae has been shown to be of order a few hundred, a number typical for the first-generation detectors under 
construction [8]. Apart from the excess signal and its time profile, additional information on the energy, direction or neutrino species is unlikely to be reconstructed in such telescopes, in contrast to dedicated supernova experiments.

The AMANDA-detector, operating in low background, sterile Antarctic ice, is at an advantage as far as detection of supernova bursts is concerned. The background counting rates in the OMs are dictated by the dark current of the photomultiplier only. They are reduced by over an order of magnitude compared to natural sea-water because of the absence of bioluminescence and radioactive decay of potassium [9]. Recently, a second advantage of using polar ice has been revealed. With the first calibration measurements it has become evident that the absorption length of light for in-situ South Pole ice is larger than what had been anticipated [10,11]. The absorption length of deep South Pole ice has the astonishingly large value of $\sim 310 \mathrm{~m}$ for the 350 to $400 \mathrm{~nm}$ light to which the PMTs are sensitive. A value of only $8 \mathrm{~m}$ had been anticipated from laboratory measurements. For supernovae neutrino signals the detector volume scales linearly with the absorption length. This can be demonstrated from a simple back-of-the-envelope estimate of the effective detection volume of an $\mathrm{OM}$ for $\mathrm{MeV}$-positrons.

The track-length of a $20 \mathrm{MeV}$ positron in ice is roughly 12 centimeters and therefore over 3000 Čerenkov photons are produced. This number combined with a typical quantum efficiency of $25 \%$ leaves $N_{\gamma} \simeq 800$ detectable photons in each event. At large distances the probability that the OM detects a photon falls as $R^{-2}$

$$
P(R) \simeq\left(R_{d} / R\right)^{2} \quad \text { for } R>>R_{d}
$$

Here $R$ is the distance to the positron shower. $R_{d}$ is defined such that photon detection probability by the $\mathrm{OM}$ is essentially unity for distances smaller than $R_{d}$, and falls quadratically for larger distances. Its value can be estimated from the relation

$$
\pi R_{d}^{2} \sim N_{\gamma} A
$$

where $A$ is the photocathode area of the OM. The relation states that the effective area for guaranteed detection is proportional to the brightness of the source and to the photocathode 
area. We next calculate the effective volume $V_{\text {eff }}$ associated with each OM by integrating the probability function over volume:

$$
V_{\mathrm{eff}} \simeq \int_{0}^{R_{\mathrm{abs}}} P(R) R^{2} d R
$$

The integral is, of course, cut off by the absorption length $R_{\mathrm{abs}}$ of the light. It is easy to see that its value is dominated by $R>R_{d}$ and negligible for $R<R_{d}$. Therefore

$$
V_{\mathrm{eff}} \simeq \int_{R_{d}}^{R_{\mathrm{abs}}} P(R) R^{2} d R \sim \frac{1}{\pi} N_{\gamma} A R_{\mathrm{abs}}
$$

for $R_{\mathrm{abs}} \gg R_{d}$. This estimate can be made more quantitative [8], but is sufficient to demonstrate that the effective volume for supernova positrons, corresponding to each OM, is proportional to both the collection area of the $\mathrm{OM}$ and the attenuation length of the light. We will demonstrate that the discovery of very large values of $R_{\text {abs }}$ for natural polar ice implies that the AMANDA detector can function as a galactic supernova watch.

At present, four AMANDA strings have been deployed in Antarctic ice at the South Pole. Each string holds 20 OMs separated by 10 meters at depths between 800 and 1000 meters. The calibration measurements have revealed absorption lengths of light for in-situ ice which are larger than what had been anticipated from laboratory experiments [10,11]. The results have been obtained by studying the propagation of Čerenkov light from high energy muons, and by studying the propagation of laser light pulsed from small nylon spheres attached to the ends of an optical fiber below each OM. The results indicate that the propagation of photons in the ice is consistent with a diffusive process with very large absorption lengths. The diffusive character of the process apparently results from scattering of the light by residual bubbles trapped in the ice. The number density of such bubbles is observed to decrease with depth. Direct measurements on ice cores indicate that such bubbles totally disappear at 1-1.5 km depth, depending on location. Clearly these results have important implications for supernova neutrino detection because we expect a linear enhancement of the effective volume per OM with absorption length.

We have calculated the effective volume of the present AMANDA detector for supernova neutrinos, taking into account the increased attenuation length, and have also considered 
the effect of scattering of the light by residual air bubbles at $1 \mathrm{~km}$ depth. The above back-ofthe-envelope estimate yields an effective volume $V_{\text {eff }} \sim 2000 \mathrm{~m}^{3}$ for an AMANDA OM with collecting area $A=0.028 \mathrm{~m}^{2}$ and an absorption length $R_{\text {abs }} \simeq 300 \mathrm{~m}$. The effective radius of each OM for detecting supernova neutrinos is about 7 meters. This is very encouraging and indicates that a single OM is similar in collection volume to the Kamiokande and IMB detectors.

We have confirmed and sharpened this result with a complete Monte Carlo simulation. Positron showers were generated using a $4 \mathrm{MeV}$ Boltzman distribution multiplied by $E^{2}$ in order to represent the increased detection efficiency associated with the rise of the neutrino interaction cross section with energy [7]. We used an electromagnetic shower Monte Carlo which was originally designed to calculate radio pulses in ice and correctly describes showers down to MeV energies [12]. The output, in the form of Čerenkov photon distributions radiated by positron showers, was fed into a simulation of the AMANDA detector. This program treated in detail both the propagation of the photons, their absorption and scattering, and the efficiencies of the OMs. For the current AMANDA configuration, with 73 stable OMs, the simulations reveal an effective volume per $\mathrm{OM} V_{\text {eff }} \simeq 1.7 \pm 0.410^{3} \mathrm{~m}^{3}$, compatible with our estimate and more than an order of magnitude above the results previously obtained assuming the properties of laboratory ice [8]. The observable signal is totally dominated by events where a positron shower yields a single photoelectron hit in a single OM. The quoted error is statistical. Although the absorption length at the peak acceptance of the OMs is well measured, its dependence on color in the near UV region is poorly determined. More precise future measurements may increase or decrease our result but cannot modify it significantly.

We have verified by Monte Carlo that the effect of residual bubbles on photon propagation is within our statistical error. This is to be expected because our results imply that the photons are collected over a radius of 7.4 meters, a distance much shorter than the absorption length of the light. For a scattering length $R_{s}$ of order 0.25 meters [10,1] photons within this radius will reach the PMT whether scattered or not. The scattering is irrelevant as long 
as the actual distance travelled by the scattered photons $\left(N R_{s}\right.$, with $\mathrm{N}$ the number of times that the photon scatters), is shorter than $R_{\text {abs }}$. For a random walk the number of scatters $N$ for a photon originating a distance d from the OM is given by

$$
N \simeq d^{2} / R_{s}^{2}
$$

The proportionality factor depends on the angular distribution of the scattering process; it is $4 / 3$ in the present problem [10,11]. The condition that the pathlength $N R_{s}<R_{\text {abs }}$ becomes

$$
d<\sqrt{\frac{4}{3} R_{s} R_{\mathrm{abs}}}
$$

which is satisfied for all distances $d$ up to 7.4 meters for $R_{s} \simeq 0.25 \mathrm{~m}$ and $R_{\text {abs }} \simeq 310 \mathrm{~m}$.

These results can be used to rescale SN1987 observations to a supernova at a distance of $d_{\mathrm{kpc}}$. To rescale the data from the Kamiokande(IMB) experiment to the AMANDA detector we need the ratio of their effective volumes corrected for the different thresholds of the instruments. This introduces a correction factor $f$ which is evaluated from the FermiDirac energy distribution and the $E^{2}$-dependence of the cross section. Using a supernova temperature for SN1987A of $4.0 \mathrm{MeV}$ [5] $f$ is about 0.8 (0.2) for Kamiokande (IMB) because of the reduced threshold of AMANDA operating as a pure counting detector. From the 11 events observed in the 2.14 kton Kamiokande detector we predict:

$$
N_{\text {Events }} \sim 11 N_{M}\left[\frac{\rho V_{\text {eff }}}{f 2.14 \text { kton }}\right]\left[\frac{52 \mathrm{kpc}}{d_{\mathrm{kpc}}}\right]^{2}
$$

For the present AMANDA configuration with $N_{M}=73, \rho=0.924 \mathrm{~g} \mathrm{~cm}^{-3}$, the density of ice, and $V_{\text {eff }}=1700 \mathrm{~m}^{3}$, we obtain 270 events per OM for a total of nearly 20,000 events.

We must now require a meaningful detection of this signal in the presence of the continuous background counting rate of all phototubes. Over the 10 seconds duration of the neutrino burst, the RMS-fluctuations of the combined noise from all the OMs is:

$$
\sigma_{1 p . e .}=\sqrt{(10 \mathrm{sec}) \nu_{1 \text { p.e. }} \mathrm{N}_{\mathrm{M}}}
$$


Here $\nu_{1 p . e .}$ represents the average background counting rate in a single module at the 1 photoelectron level, and equals1.86 kHz for the AMANDA OMs. The probability that this noise fakes a supernova signal can be, at least theoretically, estimated from Poisson statistics. The expected rate of supernova explosions in our galaxy is about $2 \times 10^{-2} \mathrm{y}^{-1}$. If the detector is to perform as a supernova watch we must require that the frequency of fake signals is well below this rate. The signal should therefore exceed $n_{\sigma} \geq 6$ which corresponds to a probability of $9.9 \times 10^{-10}$. The corresponding number of 10 second intervals does indeed exceed a century. This requirement can be relaxed if we just demand that the detector can make a measurement in the presence of independent confirmation. This may be achieved by operating parts of a single instrument as independent detectors.

For an average noise rate of $1.86 \mathrm{kHz}$ the RMS fluctuation of the 1.36 million hits expected in an interval of ten seconds is 1165. This yields a detected excess of $17 \sigma$ for a supernova at the center of the galaxy. Demanding a $6 \sigma$ signal for a supernova watch, the present AMANDA configuration with appropriate triggering, would view a radius of $17 \mathrm{kpc}$, thus covering our galaxy. Clearly signal-to-noise is proportional to $\sqrt{N_{M}}$ and therefore the number of OMs needed for fixed signal-to-noise ratio scales with the distance to the fourth power. A next-generation detector with 6000 OM's would provide a sharp signal for a supernova within a sphere of radius $52 \mathrm{kpc}$.

The results are extremely encouraging and an effort should be made to find the optimal trigger. In a realistic detector the background counting rate does not follow a Poisson distribution with a width given by simple statistics. The actual performance of the present AMANDA data acquisition relevant to the detection of supernovae is described elsewhere [13]. The AMANDA detector, frozen in then Antarctic ice, can act for decades as a supernova watch with minimal maintenance or investment in manpower and operation of the instrument. 


\section{ACKNOWLEDGMENTS}

This work was supported in part by the University of Wisconsin Research Committee with funds granted by the Wisconsin Alumni Research Foundation, in part by the U.S. Department of Energy under Grant No. DE-FG02-95ER40896, in part by the Xunta de Galicia under contract XUGA-20604A93 and in part by and the CICYT under contract AEN930729. 


\section{REFERENCES}

[1] K. Hirata, T. Kajita, M. Koshiba et al., Phys. Rev. Lett. 58 (1987) 1490; Phys. Rev. D38 (1988) 448.

[2] R.M. Bionta, G. Blewitt, C.B. Bratton, D. Casper, Phys. Rev. Lett. 58 (1987) 1494.

[3] M. Aglietta, G. Badino, G. Bologna, et al., Phys. Rev. Lett. 58 (1987) 1490; Europhys. Lett. 319 (1987) 136.

[4] C.B. Bratton et al., Phys. Rev. D37 (1988) 3361.

[5] H.A. Bethe, Rev. Mod. Phys. 62 (1990) 801.

[6] A. Burrows, Annu. Rev. Nucl. Part. Sci. 40 (1990) 181.

[7] D.N. Schramm, Comments Nucl. Part. Phys. 17 (1987) 239.

[8] F. Halzen, J.E. Jacobsen and E. Zas, Phys. Rev. D49 (1994) 1758.

[9] C. Pryor, C. E. Roos and M. S. Webster, Astrophysical Journal 329 (1988) 335.

[10] P. Askebjer, et al., Science 267 (1995) 1147.

[11] S. Tilav et al., in Proc. of the 24th Int. Cosmic Ray Conference, Rome (1994).

[12] E. Zas, F. Halzen and T. Stanev, Phys. Rev. D45 (1992) 362.

[13] R. Wisniewski et al., in Proc. of the 24th Int. Cosmic Ray Conference, Rome (1994). 